\title{
Synthetic Light-Curve Analysis of a Short Period Binary System YY Eri
}

\author{
Warisa Pancharoen', Wiraporn Maithong ${ }^{2}$ \\ ${ }^{1}$ Department of Science and technology, Faculty of Science and Technology, Phetchaburi Rajabhat University, Phetchaburi, \\ Thailand, 76000 \\ ${ }^{2}$ Department of Physics and General Science, Faculty of Science and Technology, Chiang Mai Rajabhat University, Chiang Mai, \\ Thailand, 50300 \\ 1 adiitya_sehgal@scmhrd.edu
}

\section{ABSTRACT}

YY Eri, the short-period binary system, is a W UMa type of the eclipsing binary system. This study using a 0.7-meter telescope with CCD photometric system in B V and R filters. It was observed at the Regional Observatory for the Public, Chachoengsao, Thailand on December 5, 2018, UT. The MaxIm DL software was used to analyzed the images photometry to produce the light curve. The Wilson-Devinney technique was computed the synthetic light curve that prefer to the physical properties of the YY Eri. The results show that the effective temperature of the primary and secondary star was 5533 and $5598 \mathrm{~K}$, respectively. The inclination is 81.450 and the mass ratio is 0.55 . The degree of contact was calculated as $16.64 \%$

Article Received: 10 August 2020, Revised: 25 October 2020, Accepted: 18 November 2020

\section{Introduction}

Only around half of the stars that we see when we look to the sky are single stars. The rest are multiple systems, consisting of two or more stars orbiting around each other due to their gravitational attraction. Two stars orbiting each other are called binary stars. Measurements of the dynamical interaction of the components in eclipsing binary stars provide the most accurately determined parameters of stars (Penélope,2015). The Uma binary star are divided into two subtypes $\mathrm{W}$ and A. W UMa variables have primary and secondary eclipses of nearly equal depths. The spectral type of subtypes A-type W UMa systems are A-F spectra, and G-K spectral for W-type systems. The period of subtypes A-type W UMa is 0.4 to 0.8 day and 0.22 to 0.4 day for subtypes $\mathrm{W}$ type W UMa (Binnendijk,1965)

The short-period binary system YY Eri is a Wsubtype of W UMa type which R.A. 04h $12 \mathrm{~m}$ $08.849 \mathrm{~s}$ and Dec. $-10^{0} 28^{\prime} 09.993$. The light curve of YY Eri have pattern of variation, with a slightly asysmestry (E. Budding,1997). The mean magnitude of YY Eri is 8.4, a spectral type G5V and the period is 0d.32149510 (C.Maceroni,1994.). Radius velocity of YY Eri is $-15 \mathrm{kms}^{-1}$ (R. Nesci,1986.). The value of masses

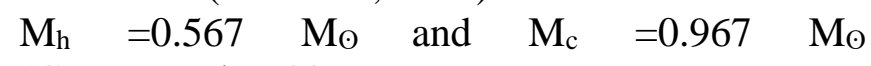
(C.Maceroni,1982.).
For this work, we use the Wilson-Devinney Technique to considers about the physical properties of YY Eri Uma binary star.

\section{Experimental}

YY Eri was observed 5 December 2018, UT at the Regional Observatory for the Public, Chachoengsao, Thailand. The 0.7 - meter reflecting telescope and $\mathrm{CCD}$ with the blue (B), standard visual (V) and red (R) filters of the UBV system were used. The figure 1 showed a sample of the YY Eri image. In this work, TYC 5315986-1 and HD 26650 were chosen as comparison star and check star. The information of them were shown in table 1. The YY Eri images were analyzed by photometry technique with MaxIm DL program. The Wilson-Devinney Technique was used to calculate the properties of this binary system. 


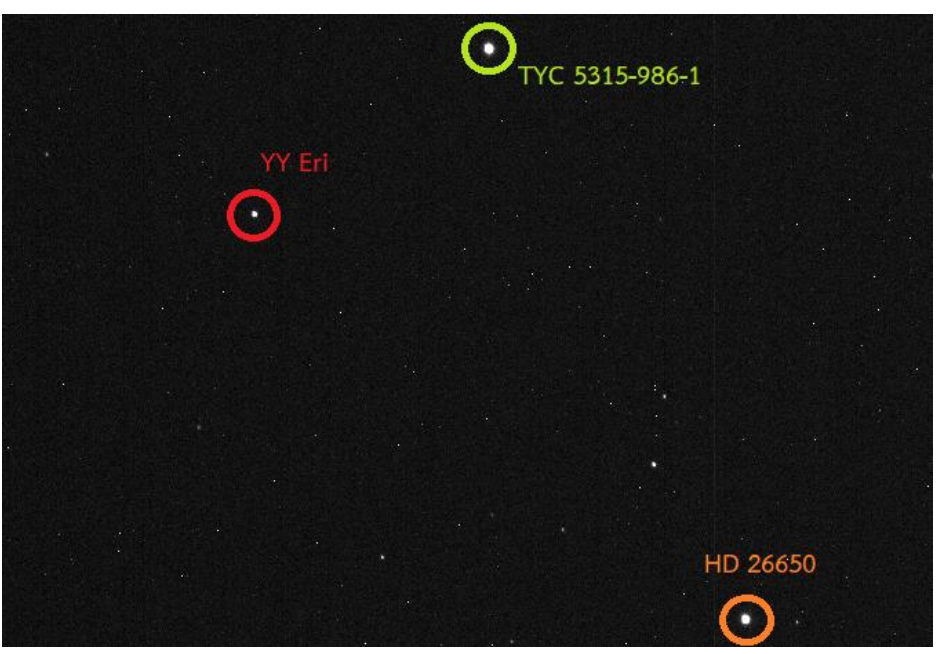

Figure 1. The image of YY Eri.

Table 1. The basic information of the YY Eri from this observation.

\begin{tabular}{lllll}
\hline Star & $\begin{array}{l}\text { R.A.(h } \\
\mathrm{m} \mathrm{s})\end{array}$ & $\begin{array}{l}\text { Dec. } \text { ( }^{\mathrm{o}} \\
\text { ') }\end{array}$ & Magnitude \\
\hline YY Eri & $04 \quad 12$ & $-10 \quad 28$ & 8.41 \\
& 08.849 & 09.993 & \\
TYC & $04 \quad 12$ & $-10 \quad 26$ & 11.02 \\
$5315-$ & 21.364 & 03.797 & \\
$986-1$ & & & \\
HD & $04 \quad 12$ & $-10 \quad 33$ & 8.43 \\
26650 & 32.596 & 57.865 & \\
\hline
\end{tabular}

The W-subtype binary system is the mass of a secondary star less than a primary star. So, we analyzed the best of the mass ratio $\mathrm{q}=\mathrm{m}_{2} / \mathrm{m}_{1}$ from $0.20,0.30,0.40,0.50,0.60 .0 .70,0.80,0.90$ and 1.00. The relation of the computation error $(\Sigma)$ and the mass ratio as shown in Fig.2. The best error of the mass ratio from Fig. 2 was 0.555882 . This value was used for the initial value to calculate the physical properties of YY Eri.

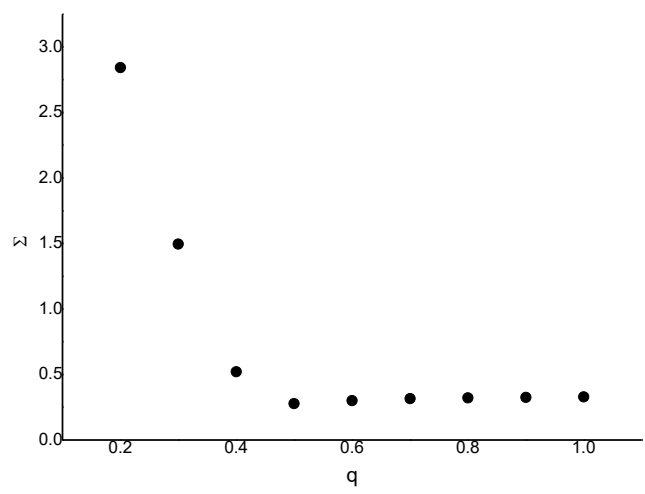

Figure 2. Variance of the mass ratio $q$ of the YY Eri

\section{Results and Discussion}

The synthetic light curve of YY Eri, as shown in Figure 3, was constructed from photometric data computation using Wilson-Devinney software. The dot is data from the observation and the red line is data from calculated. The light curve shown that the YYEri is the W UMa type of the eclipsing binary system. The best solution of the YY Eri, as shown in Table 2. The inclination of orbital is $81.45^{\circ}$ corresponding to R Nesci et.al.(1985), Y. Yang and Q. Liu (1999). The parameter mass ratio $\mathrm{q}=0.55582$, the surface temperature of primary star $\left(\mathrm{T}_{1}\right)$ is $5533 \mathrm{~K}$ and the surface temperature of secondary star $\left(\mathrm{T}_{2}\right)$ is $5598 \mathrm{~K}$ nearly the results of R Nesci et.al.(1985), Y. Yang and Q. Liu(1999), shown that YY Eri are G5 spectral. The gravity of darkening coefficient of primary and secondary star $\left(g_{1}\right.$ and $\left.g_{2}\right)$ are equal to 0.32 same to data from Y. Yang and Q. Liu(1999). We see that the value of the gravity of darkening coefficient $(\mathrm{g})$ and the bolometric albedos (A) indicated that the YY Eri are convective envelope stars. Degree of contact of the binary star is $16.64 \%$.

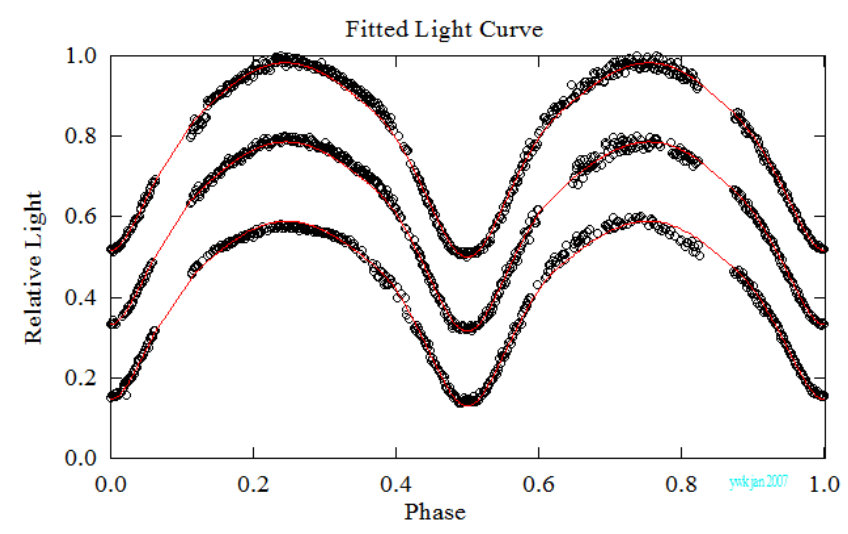

Figure 3. Synthetic light curve of the YY Eri.

Table 2. Parameter for simulate the YY Eri Model.

\begin{tabular}{ll}
\hline Parameter & The Best Solution \\
\hline $\mathrm{i}$ & $81.45^{0} \pm 0.10$ \\
$\mathrm{~g}_{1}$ & 0.32 \\
$\mathrm{~g}_{2}$ & 0.32 \\
$\Omega_{1}=\Omega_{2}$ & $2.927108 \pm 0.008878$ \\
$\Omega_{\text {in }}$ & 2.981722 \\
$\Omega_{\text {out }}$ & 2.653566 \\
$\mathrm{~T}_{1}(\mathrm{~K})$ & $5533 \pm 154$ \\
$\mathrm{~T}_{2}(\mathrm{~K})$ & $5598 \pm 146$
\end{tabular}




\begin{tabular}{ll}
\hline $\mathrm{A}_{1}$ & 0.50 \\
$\mathrm{~A}_{2}$ & 0.50 \\
$\mathrm{q}$ & $0.555882 \pm 0.005128$ \\
$\mathrm{~L}_{1} /\left(\mathrm{L}_{1}+\mathrm{L}_{2}\right)_{\mathrm{B}}$ & $0.60955 \pm 0.02696$ \\
$\mathrm{~L}_{1} /\left(\mathrm{L}_{1}+\mathrm{L}_{2}\right)_{\mathrm{V}}$ & $0.61290 \pm 0.02520$ \\
$\mathrm{~L}_{1} /\left(\mathrm{L}_{1}+\mathrm{L}_{2}\right)_{\mathrm{R}}$ & $0.61602 \pm 0.02520$ \\
Degree of Contact & 16.64 \\
$(\%)$ & \\
\hline
\end{tabular}

The physical properties value from Table 2 were forecasted its binary system model, as shown in Figure 4.

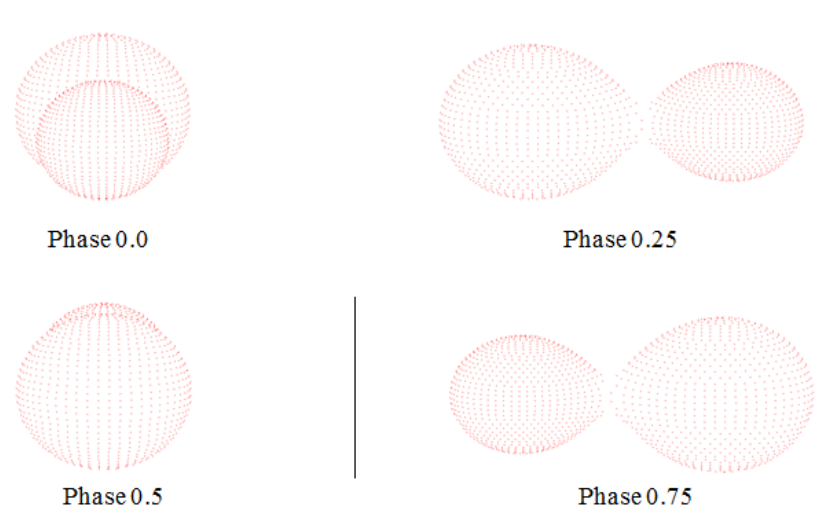

Figure 4. YY Eri model.

\section{Conclusion}

The eclipsing binary system YY Eri was observed on December 5, 2018, UT at the Regional Observatory for the Public, Chachoengsao, Thailand and was analyzed at Faculty of Science and Technology, Chiang Mai Rajabhat University, Chiang Mai and Phetchaburi Rajabhat University, Phetchaburi, Thailand. In this research, the physical properties were computed by WilsonDevinney technique. The solution shows that the binary system YY Eri is a W-type contact binary which a mass ratio is 0.555882 and the degree of contact is 16.64 percents.

\section{Acknowledgment}

The author would like to acknowledge the National Astronomical Research Institute of Thailand (Public Organization) for this observation.

\section{References}

[1] C. Macerini,L. Milano and G. Russo.,(1982). Determination of parameters of W Uma systems.III:CC
Com, YY Eri, V502 Oph and TY Pup., astronomy and astrophysics supplement series.,49.123-128.

[2] C.Maceroni,O.vilhu,F Van't Veer,W Van Hamme.(1994).Surface imaging of latetype contact binaries I :AE Phoenicis and YY Eridani., astronomy and astrophysics.,288.,529-537.

[3] E. Budding. Et. Al.,(1997). The contact binary system YY Eri., Astrophysics and space science.,246.,229-242.

[4] F.H. Shu, S.H. Lubow and L. Anderson, (1976). On the structure of contact binaries. I - The contact discontinuity., Astrophysics Journal, 209, 536.

[5] J.M. Kreiner, (2004). An Atlas of O-C Diagrams of Eclipsing Binary Stars., Acta Astronomica, 54, 207-210.

[6] L.B. Lucy, (1976). W Ursae Majoris systems with marginal contact., Astrophysics Journal, 205, 208-216.

[7] L. Binnendijk, Veroeffentlichungen der Remeis-Sternwarte zu Bamberg, Nr. 40., p. 36,1965

[8] Penélope Alejandra Longa-Peña.,(2015). Orbital Parameters Estimation for Compact Binary Stars. Doctor of Philosophy. Department of Physics.University of Warwick.

[9] R.E. Wilson and E.J. Devinney, (1971). Realization of Accurate Close-Binary Light Curves: Application to MR Cygni., Astrophysics Journal, 166, 605-619.

[10] SIMBAD Astronomical Database - CDS (Strasbourg) http://simbad.ustrasbg.fr/simbad/

[11] R. Nesci,C. Macerini,I. Milano and G. Russo,(1986).YY Eri revisited., astronomy and astrophysics.,159.,142-146

[12] Y. Yang and Q. Lui.,(1999).Photometric study of the late-type contact binary YY Eridani., astronomy and astrophysics supplement series.,136.,139-143. 